\title{
LAPAROSCOPIC ASSISTED ANORECTOPLASTY: A NEW DEFINITIVE REPAIR OF HIGH IMPERFORATE ANUS
}

\author{
Tarek A Gobran, Ameen Saleh, Omar A ElEkiabi \\ Unit of Pediatric Surgery, Zagazig University
}

\begin{abstract}
Background/ Purpose : The classic treatment of the the high anorectal anomalies over the last 3 decade is the posterior sagittal anorectoplasty. . Laparoscopic assisted anorectoplasty is a minimally invasive technique that aims at preserving the sphincters, placing sensate skin within the control of the sphincters, and shortening recovery. The objective of this work is to evaluate the effectiveness of the laproscopic assisted anorectoplasty for the infants suffering from high imperforate anus.

Materials \& Methods: Twenty eight patients with high anorectal anomalies were included in this study. All patients had laparoscopically assisted anorectal pull-through (LAARP) for high imperforate anus. Hospital charts and surgical notes were reviewed and clinical characteristics tabulated.

Results: Postoperative complications included rectal mucosal prolapsed $(n=3)$, intestinal obstruction $(n=1)$, anal stricture $(n=1)$, and development of blind ended posterior urethral fistula $(n=1)$. There were no complications related to the urinary system, also there was no wound infection, no hernia through

the port sites, or anal retraction. Six cases developed constipation. The overall clinical assessment for continence of the cases showed that $50 \%$ had good outcome, $36 \%$ had moderate, and $14 \%$ had poor functional outcome

Conclusion: LAARP for treatment of high anorectal anomalies is a feasible technique with encouraging functional results. Before recommendation of its use as a treatment of choice, we recommend more studies for further evaluation of this technique, as the studies done for this subject are still few.
\end{abstract}

\section{INTRODUCTION}

T he posterior sagittal anorectoplasty (PSARP) has been the most commonly used technique for the repair of high and intermediate anorectal anomalies since the early $1980 \mathrm{~s}^{\mathbf{( 1 )}}$.

With the advent of minimally invasive surgery, Georgeson et al. in 2000 published their first results with laparoscopic assisted anorectal pullthrough (LAARP) ${ }^{(2)}$. Since then, several reports on LAARP have been published ${ }^{(3-7)}$.

Georgeson et al. ${ }^{(2)}$ mentioned that LAARP has the advantages that it utilizes minimal perineal dissection, allowing excellent visualization of the fistula and the surrounding structures, avoid cutting through pelvic floor muscles, preservation of the distal rectum, and accurate placement of the rectum within the levator ani and external anal sphincter muscle complex. These facts may suggest better functional results over the classic PSARP.

However, still, there is a significant debate about the functional outcome of LAARP in comparison with the open surgical repair as mentioned before $^{(5,8,9)}$.

Hozaim et al. ${ }^{(10)}$ reviewed all the published literatures about the laparoscopic treatment of anorectal anomalies, written in English over the period from June 2000 till April 2008, and they concluded that, the number of studies dealing with LAARP is low , and there is a need for both a standardization and improvement in the quality of reporting in LAARP research, which will ultimately allow for evidence-based surgical decision making.
Considering that this subject is still a matter of debate, the aim of our study is to add more research work for evaluation of the effectiveness of LAARP for correction of high anorectal anomalies, regarding the technique and the outcome

\section{PATIENTS AND METHODS}

A prospective clinical study, included twenty eight patients with high anorectal anomalies, presented to the pediatric surgical unit, Zagazig University from January 2012 to January 2014. All patients had laparoscopically assisted anorectal pull-through (LAARP) for high imperforate anus. Hospital charts and surgical notes were reviewed and clinical characteristics tabulated. All patients underwent divided sigmoid colostomy at birth as an initial step. Patients had distal colostograms before anorectoplasty to define the site of communication of rectal fistulae with genitourinary structure

\section{Operative procedure:}

Pneumoperitoneum is created with $\mathrm{CO}_{2}(8-12$ $\mathrm{mm}$ of $\mathrm{Hg}$ ) by the open method. The abdomen is accessed by three ports, one $5 \mathrm{~mm}$ umbilical port for $30^{\circ}$ telescope and two $5 \mathrm{~mm}$ accessory working ports one at right lumbar region and another at left hypochondrium. For comfort, an additional $5 \mathrm{~mm}$ suprapubic port or a stay suture will help retraction of the bladder . The bladder, despite being decompressed by urinary catheter, is retracted anteriorly by a transcutaneous bladder stitch. A small window is first developed in the mesorectum at the level of the peritoneal reflection with an electrocautery. The rectum is mobilized beginning posteriorly and proceeding 
laterally and anteriorly in a circumferential fashion. The terminal branches of the sigmoid and superior rectal arteries are divided, if needed, to gain adequate length. Caudal dissection at this step should not be too close to the bowel to avoid colotomy, and not too far away to avoid nerve or ureter injury. As the fistula is visualized, it is dissected free, and clipped . At this step the prostate, seminal vesicles, and vas deferens are liable to injury and care is required. The rectum is divided between the clips (Fig.1 ).

Care is taken to secure the proximal bowel at this step to safeguard against soiling of the operating field. The fistula closure may be reinforced by an endoloop. Another method to close the fistula is by using intra corporeal transfixion. A third method of fistula ligation is by passing a loop from the perineum up around the fistula then performing an extra corporeal knot tying. Puborectalis sling is identified before fistula division, traction on the fistula helps in midline dissection to create the pull through channel. With cephalad traction on the fistula, both bellies of pubococcygeus can easily be identified in relation to the urethra (fig.2).

The classic anatomic arrangement of the puborectails, resembling a "sling-shot," can often be appreciated. The contractility of the levator ani muscle and center of its two bellies is identified by the conventional diathermy in a low setting current, which can be used just as effectively as a laparoscopic muscle stimulator as advised by Karthik et al. ${ }^{(11)}$

When there is insufficient muscle mass to clearly ascertain the pubococcygeus, the midline is identified based on the position of the distal end of the divided fistula and the urethra. Externally, the anal area of the perineum is mapped out using transcutaneous electro stimulation (muscle stimulator with 100-150 milliamps current). The area of maximal contraction and ventro-cephalad elevation of the perineum is noted with simultaneous contraction of the puborectalis. The anterior and posterior limits of this anal area are marked and a $12 \mathrm{~mm}$ vertical midline incision is made at the proposed anal orifice.

The Veress needle was first inserted through the puborectalis sling under laparoscopic vision, followed by the guide wire ( as described by Barbary et al. ${ }^{(12)}$, then the dissected intrasphincteric plane is dilated with serial Hegar's dilators up to $10-12 \mathrm{~mm}$ size railroaded over the suction canula between the two bellies of the pubococcygeus muscle in the midline, under vision abdominally (Fig.3 ).

A $10 \mathrm{~mm}$ trocar is inserted through dilated tract into peritoneal cavity. The divided rectal fistula is grasped using an endo-Babcock clamp and pulled onto the perineum through the newly created tract taking care not to twist the bowel. Anoplasty is done with 4-0 vicryl stitches. Finally the rectum is retracted cephalad. This retraction lengthens the skin lined anal canal .

Pelvic MRI and EMG of the external anal sphincter are done for each baby to assess the position of the rectum in relation to the muscles, and the degree of muscle hypoplasia present. This was done for each patient before closure of colostomy .Evaluation for fecal continence was done two months following colostomy closure. Each mother was asked to record a defecation dairy for a period of 7 days. Items for recording include: the day, time, amount of stool (large, average, small, or staining of clothes), and the consistency of stool either solid or liquid. Patients were classified, according to Lin et al. ${ }^{(\mathbf{1 3})}$, into the following groups. Good: low number of motions per day (1-3) with large volume of stool per motion, the patient is clean between motions, with no staining. Moderate: moderate frequency of motions daily (4-6) and infrequent staining between motions. Poor: frequent motions per day 7 and more, with small volume of stool per motion with frequent staining of clothes between motions. 


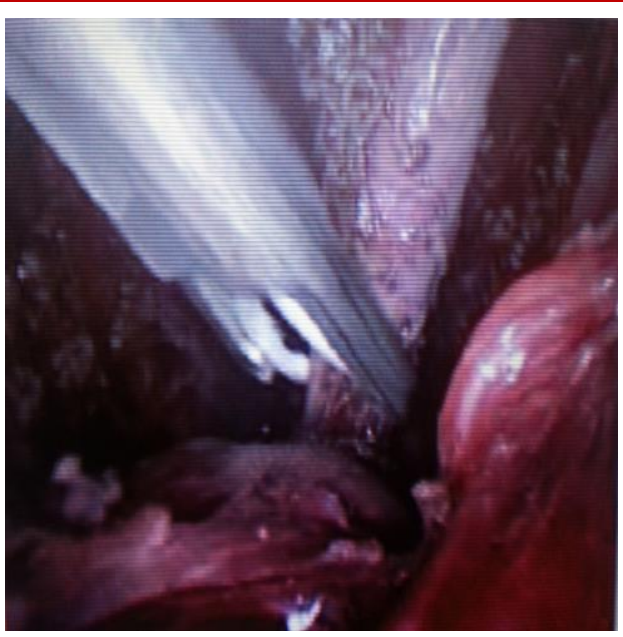

Figure (1): Clipping the fistula

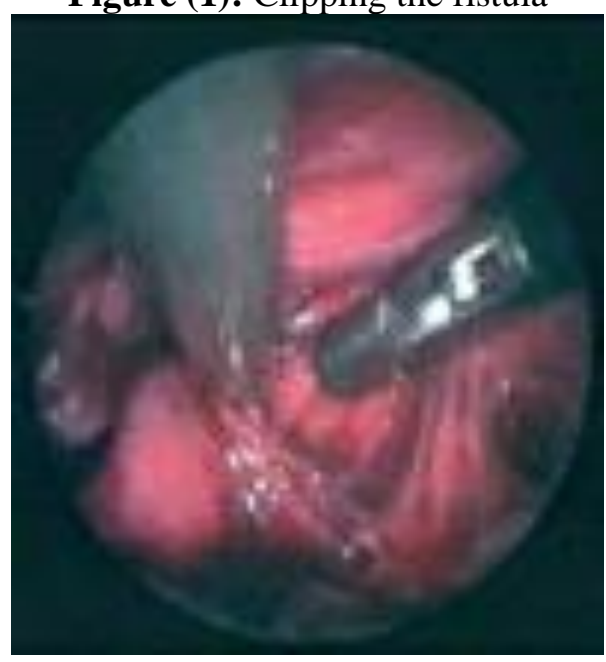

Figure (2): Pubococcyeus muscle

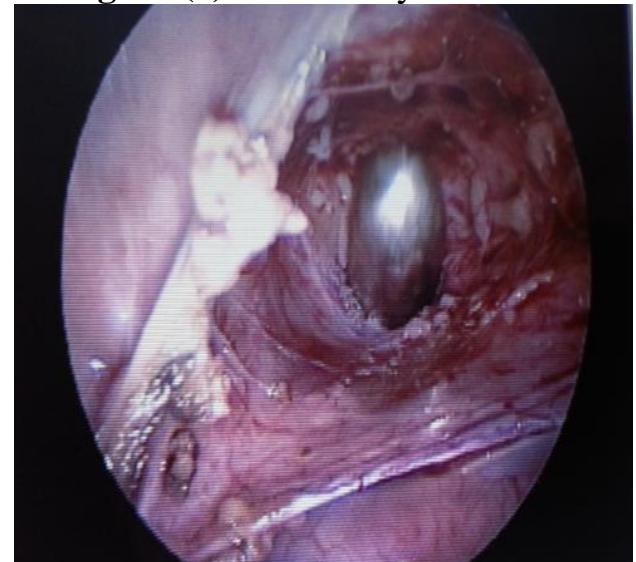

Figure (3): Hegar dilatation of pullthrough canal

\section{RESULTS}

Laparoscopically assisted anorectoplasty (LAARP) was done for 28 patients; 24 males and 4 females. The age at surgery ranged between 2 and 12 months; mean 7 months. Fourteen patients had rectoprostatic fistula, four patients had rectobulbar fistula, five had recto-bladder neck fistula, four recto vaginal, one had anorectal agenesis without fistula .
Post operative complications occurred in six patients; three developed rectal mucosal prolaps, that required mucosectomy before colostomy closure. One patient had adhesive intestinal obstruction, which necessitated a laparotomy for adhesiolysis

three weeks later. One patient had anal stricture, and one patient had a posterior urethral blind ended fistula diagnosed by VCU, but not a true urethral diverticulum ( fig.4). 
There were no other complications related to the urinary system such as retention of urine, urinary incontinence, urethral stricture, or recurrence of recto urogenital fistula. Also there was no wound infection, no hernia through the port sites, or anal retraction. Table 1 summarizes the postoperative complications.

Result of Pelvic MRI: In 24/28 patients the rectum was centralized in the three levels of transverse sections at levator ani, muscle complex, and external anal sphincter (fig.5). In all the 28 patients there was no urethral diverticulum. In one patient the rectum was mal-positioned in the lower most level where it was anterior in relation to the external anal sphincter. This patient had later limited anoplasty to push back the rectum within the external sphincter. In 3 more patients the rectum was deviated to the one side at the level of the muscle complex (Fig.6).

EMG study showed that 25 patients had preserve integrity of the EAS, two patients had no activity at 12 o'clock, and one patient had no activity at 6 o'clock, and the muscle was disturbed at this site. Evaluation: Constipation developed in 6 patients , however it responded well for laxatives and bowel habit training programs in the all cases. The overall clinical assessment for continence of the cases showed that $50 \%$ had good outcome, $36 \%$ had moderate, and $14 \%$ had poor functional outcome. Table 2 summarizes the state of continence in different types of fistula

Table (1): The postoperative complications

Complication

Rectal prolapse

Adhesive intestinal obstruction

Blinded end urethral fistula

Urinary incontinence

Wound infection

Hernia in port site

Anal stenosis

Total
No of patients

3

1

1

0

0

0

1

6

Table (2): Results of continence after LAARP in different types of fistulae according to Lin criteria in 2003

\begin{tabular}{|c|c|c|c|c|}
\hline Type of the fistula & Number & Good & Moderate & Poor \\
\hline Vesical & 5 & $2(40 \%)$ & $2(40 \%)$ & $1(20 \%)$ \\
\hline Prostatic & 14 & $8(57 \%)$ & $4(29 \%)$ & $2(14 \%)$ \\
\hline Bulbar & 4 & $2(50 \%)$ & $2(50 \%)$ & 0 \\
\hline Vaginal & 4 & $2(50 \%)$ & $1(25 \%)$ & $1(25 \%)$ \\
\hline Non & 1 & 0 & $1(100 \%)$ & 0 \\
\hline Total & 28 & $14 \quad(50 \%)$ & $10(36 \%)$ & $4(14 \%)$ \\
\hline
\end{tabular}




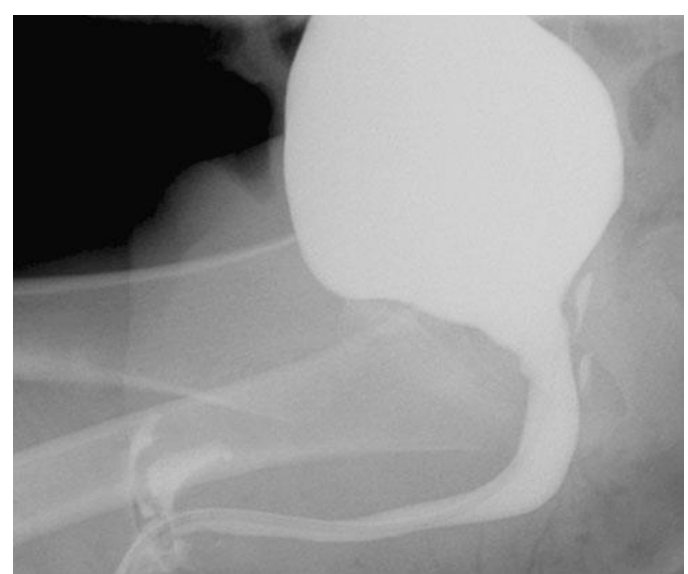

Figure (4): VCU showing an Blinded end posterior urethral fistula

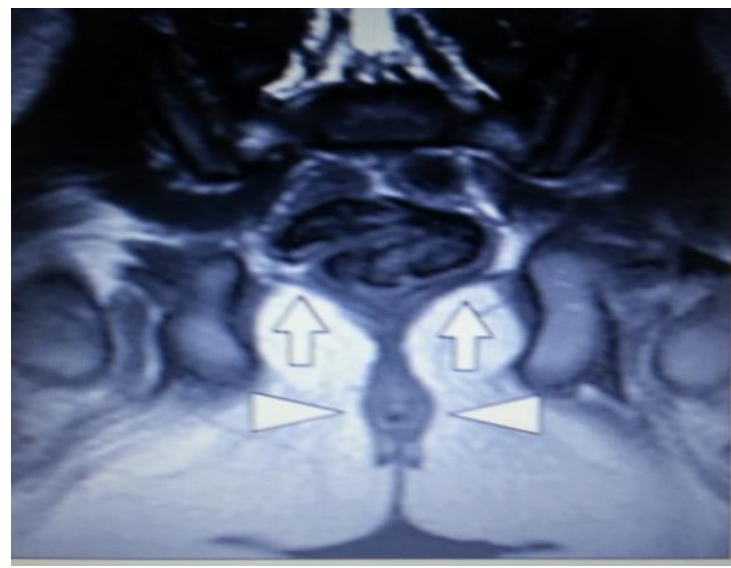

Figure (5): MRI showing a centralized rectum in pelvic floor

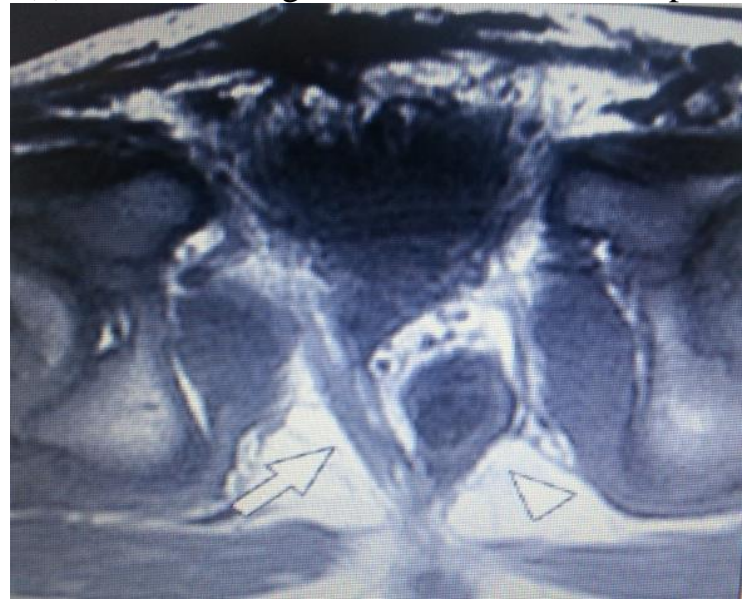

Figure (6): MRI showing displacement of the rectum to the right side of muscle complex

\section{DISCUSSION}

The PSARP involves incision from coccyx to perineal body, to widely expose the external sphincter, the levators, the rectum, and distal fistula to facilitate surgical repair ${ }^{(\mathbf{1})}$.

It is possible that dividing the sphincters during dissection and lining the anal canal with insensate mucosa negatively influence continence. Georgeson et al. ${ }^{(2)}$ described a new surgical repair for high imperforate anus that utilizes a laparoscopic technique to reduce the amount of posterior dissection required for accurate placement of the bowel into the muscle complex.
The desire to preserve the sphincters, place sensate skin within the control of the sphincters, and shorten recovery has led to the development of this minimally invasive surgical technique. Pulling the rectum in the proper anatomical site is the keystone step in the definitive reconstruction. Laparoscopic approach gives the credit of visualization of the puborectalis muscle sling. Moreover direct stimulation of this muscle can be done by passing the muscle stimulator via a 10 $\mathrm{mm}$ port, or using a diathermy at a low current as done in our study. These methods proved accurate as confirmed by the post operative MRI 
examination . In cases of recto-bladder neck fistula and high rectovaginal fistula, a laparotomy, in addition to the posterior sagittal approach is mandatory $^{(\mathbf{1 4})}$. Using the laparoscopic technique, there have been no problems in gaining enough length for the pull-through, even if the distal colonic loop is short.

Postoperative complications after LAARP occurred in six patients $(21.5 \%)$ rectal prolapse in three $(10 \%)$ and adhesive intestinal obstruction in one patient $(3.5 \%)$. One patient developed anal stricture, in this case the distal colonic loop was very short, it was pulled through by LAARP with some tension which cause some degree of ischaemia, however, the stricture improved under regular frequent dilatation. One case developed a posterior urethral blind ended fistula detected by postoperative VCU, but not an actual dilated urethral diverticulum. There have been reports ${ }^{(8,9,15,16)}$ describing the development of posterior urethral diverticula leading to compression and obstruction of the urethra as they increase in size. It was suggested that this complication occurred more often in laparoscopic procedures than in open PSARP. This complication is expected on leaving a long stump of the fistulous communication on the urethral side during division of the fistula. In our study, we did not met with this complication, we did not detect any diverticulum by VCU or MRI in suspected cases. There were no wound infection, no incisional hernia through the port opening. In this work, no intra-operative injury to genitourinary organs occurred and there were no urinary incontinence or retention of urine after catheter removal. Most of the reported urinary injuries after an imperforate anus repair in male patients, result from of dennervation or damage provoked by the operation. Some of these patients may suffer from urinary incontinence ${ }^{(17)}$.

The evaluation of the position of the rectum after pull- through is best done by MRI. Yamataka et al used the Postoperative MRI in three patients with high imperforate anus repaired by LAARP and confirmed that the rectum was located in the center of the levator ani muscle sling and muscle complex $^{(18)}$.

Wong et al performed post operative MRI for ten patients after LAARP and when compared with conventional PSARP patients, a significantly lower proportion of LAARP patients had sphincter asymmetry $(40 \%$ vs. $100 \%, \mathrm{p}<0.05)$ and peri-rectal fibrosis $(40 \%$ vs. $87.5 \%, \mathrm{p}<0.05)$. The positioning of the rectum was, however, central for both groups $(90 \% \text { vs. } 87.5 \%)^{(\mathbf{1 9})}$.

In this study post-operative MRI was done for all cases. The rectum was centralized in relation to the muscles of continence in $85.7 \%$ of patients $(24 / 28)$. One had anterior position of the rectum in relation to the EAS.

The other three had deviation of the rectum to one side of the muscle complex. In the case in which the rectum was displaced anterior to the EAS, the deformity was corrected by repositioning of the rectum using a limited posterior sagittal approach, and he developed a good degree of continence. In the 3 cases in which the rectum was displaced to one side of the muscle complex, only one case developed a poor degree of incontinence, necessitating redo repair by PSAPR under new covering colostomy, and he did well after the repair.

Constipation is a major complication in patients who have undergone posterior sagittal anorectoplasty (PSARP) for a high anorectal malformation. In 1995, Peña (20) presented a very critical and detailed analysis of 245 patients examined postoperatively. Chronic constipation occurred with a rate of $55.5 \%$ in cases of bulbar fistulae but in the higher anorectal malformations with prostatic or bladder neck fistulae the rate was $41.4 \%$ and $18.2 \%$ respectively. In a study by Chen et al. ${ }^{(21)}$, constipation was noted shortly after anorectoplasty in 18 of $25(72 \%)$ high and intermediate anomalies treated with PSARP, but in none of 5 high and intermediate anomalies treated with Rehbein's mucosa-stripping endorectal pull-through. It was concluded that anorectal function in patients with repaired imperforate anus seems to be more affected by the extent of endo-pelvic dissection.

In our twenty eight patients, 6 patients (21.4\%) developed constipation, all these cases responded well for the conservative treatment using laxatives and bowel habit training programs.

For evaluation of the state of continence in this study, we mainly relied upon the number of motions per day and the presence of soiling in between motion as described by Lin et al. ${ }^{(13)}$.

The overall result of our study as regard continence showed that $50 \%$ had good, $36 \%$ moderate and $14 \%$ poor results. Regarding the technique of LAARP the number of series in the literature is still small to draw statistical significance. However, Lin et al. ${ }^{(13)}$ used the number of motions per day for the early clinical assessment of nine cases of high imperforate anus after LAARP. Seven of nine LAARP patients had an acceptable frequency of one to four bowel openings per day.

El Barbary et al. ${ }^{(\mathbf{1 2})}$ studied 20 boys after LAARP regarding the state of continence, and he found that $55 \%$ have good results $(11 / 20), 35 \%$ moderate $(7 / 20)$ and $10 \%$ have poor results $(2 / 20)$. 
A result that comparable to some extend to our results.

On the other hand, the posterior sagittal anorectoplasty (PSARP) was used by Pena et al. ${ }^{(20)}$ in 25 patients as a primary operation. And he compared his results with the results of the classic abdomino-perineal pull through, which was done before. After the pull-through operation six out of 16 patients $(37.5 \%)$ were continent, versus $40 \%(10 / 25)$ following the PSARP. Patients with a sacral defect were continent only in $16 \%$ as compared to $44 \%$ of the patients with a normal sacrum.

It seems that LAARP has a better functional results in comparison to the open surgical methods. El Debeiky et al. ${ }^{(22)}$, in a study for comparison between the functional results of LAARP and PSARP, concluded that the state of continence with the laparoscopic technique in high anorectal malformations showed acceptable results but needs bigger series with longer followup for a proper evaluation of this technique.

The basic concept of LAARP is that of fistula transfer from the urethra through the levator sling and external anal sphincter muscle complex to the anal surface. There is no need to divide the muscle complex from below, because the pubococcygeus can be visualized and targeted from above, and immediately after the procedure, strong and symmetric contraction of the sphincter around the neoanus provides reassurance that the bowel was accurately brought through the sphincters. This approach conserves bowel, and by securing the fistula to the perineum, preserves distal bowel wall, which may contain muscle fibers of internal sphincter function. The preliminary results of LAARP are encouraging, and with increased use of laparoscopy it might be the treatment of choice in high and intermediate anorectal, but more researches in this subject are recommended before standardizing this technique.

\section{REFERENCES}

1- Devries PA. Posterior sagittal anorectoplasty: important technical considerations and new applications. J Pediatr Surg 1982; 17:796-811

2- Georgeson KE, Inge TH, Albanese CT. Laparoscopically assisted anorectal pull-through for high imperforate anus - a new technique. J Pediatr Surg 2000; 35:927-930

3- Petit T, Mure PY, Gelas T, Demarche M, Allal H, Becmeur F, Varlet F, Philippe P, Weil D, Heloury Y. Minimal invasive surgery for anorectal malformation in boys: a multicenter study. J Laparoendosc Adv Surg Tech A 2009; 19:233-235

4- Kimura O, Iwai N, Sasaki Y, Tsuda T, Deguchi E, Ono S, Furukawa T. Laparoscopic versus open abdominoperineal rectoplasty for infants with high- type anorectal malformation. J Pediatr Surg 2010; 45:2390-2393

5- Bischoff A, Levitt MA, Peña A. Laparoscopy and its use in the repair of anorectal malformations. J Pediatr Surg 2011; 46:1609- 161710.

6- De Vos C, Arnold M, Sidler D, Moore SW. A comparison of laparoscopic-assisted (LAARP) and posterior sagittal (PSAARP) anorectoplasty in the outcome of intermediate and high anorectal malformations. S Afr J Surg 2011; 49:39-43

7- England RJ, Warren S, Frederik J BeekL, Bezuidenhout L, Numanoglu A, Millar AJ. Laparoscopic repair of anorectal malformations at the Red Cross War Memorial Children's Hospital: taking stock. J Pediatr Surg 2012; 47:565-570

8- Belizon A, Levitt M, Shoshany G, Rodriquez G, Peña A. Rectal prolapse following posterior sagittal anorectoplasty for anorectal malformations. J Pediatr Surg 2005; 40:192-196

9- Alam S, Lawal TA, Peña A, Sheldon C, Levitt MA. Acquired posterior urethral diverticulum following surgery for anorectal malformations. J Pediatr Surg 2011; 46:1231-1235

10-Al-Hozaim O, Al-Maary J, AlQahtani A. Laparoscopic-assisted anorectal pull-through for anorectal malformations: asystematic review and the need for standardization of outcome reporting. J Pediatr Surg. 2010; 45(7):1500-4.

11-Bhandary K, Kumaran V, Rajamani G, Kannan S, Venkatesa Mohan N, Rangarajan R and Muthlingam V. Laparoscopic assisted anorectal pull through: Reformed techniques. J Indian Assoc Pediatr Surg. 2009; 14(4): 210-214.

12-El-Barbary MM, Fares $\mathrm{AE}$ and El-Saket $\mathrm{H}$. Laparoscopically Assisted Anorectoplasty: A New Definitive Repair of High Imperforate Anus. Annals of Pediatric Surgery 2008; 4(1): 1-7.

13-Lin CL, Wong KK, Lan LCL, Chen CC and Tarn PKH. Earlier appearance and higher incidence of the recto-anal relaxation reflex in patients with imperforate anus repaired with laparoscopicaily assisted anorectoplasty. Surg Endosc 2003; 15.

14- Keily E M, Peña A. Anorectal malformations, in O'Neill JA, Rowe MI, Grosfeld J L, et al: Pediatric Surgery. St. Louis, M O, Mosby 1998; 1445-8.

15-Bischoff A, Levitt MA, Peña A. Laparoscopy and its use in the repair of anorectal malformations. J Pediatr Surg 2011; 46:1609.

16-Vinnicombe SJ, Good CD, Hall CM. Posterior urethral diverticula: a complication of surgery for high anorectal malformations. Pediatr Radiol 1996; 26:120-126.

17-Peña A. Anorectal malformation in: Operative pediatric surgery, McGraw-Hill Company 2003; 739-44.

18- Yamataka A, Segawa O, Yoshida R. Laparoscopic muscle electrostimulation during laparoscopyassisted anorectal pull-through for high imperforate anus. J Pediatr Surg 2001; 36:1659-61. 
19-Wong KK, Khong PL, Lin SC. Post-operative magnetic resonance evaluation of children after laparoscopic anorectoplasty for imperforate anus. Int J Colorectal Dis 2004; 20.

20-Peña A. Anorectal malformations. Sem Pediatr Surg. 1995; 4:35-47.
21-Chen C C, Lin C L, Lu W T. Anorectal function and endopelvic dissection in patients with repaired imperforate anus. Pediatr Surg Int 1998; 13: 133-7.

22-El-Debeiky MS, Safan HA, Shafei IA, Kader HA, Hay SA. Long-term functional evaluation of fecal continence after laparoscopic-assisted pull-through for high anorectal malformations. J Laparoendosc Adv Surg Tech A. 2009; 19(Suppl 1): S51-4. 